\title{
The stigmata of obesity: thoughts and attitudes of adolescent school children
}

\author{
A. Hutch, M. He, C. Costello, A. M. Reilly, M. E. J. Lean, W. S. Leslie and C. R. Hankey \\ Human Nutrition Section, University of Glasgow, New Lister Building, 10-16 Alexandra Parade, G31 2ER, UK
}

A stigma is defined as: "a mark of disapproval imparted by society in which an individual is criticised as a result of a particular condition" (Puhl and Brownell, 2001). Limited evidence suggests obese and overweight adolescent school children are stigmatised by their peers, which may impede educational success, work and social development. With increasing prevalence of adolescent obesity in Europe, this study aimed to characterise the beliefs and attitudes towards obesity of school pupils aged 16-18 years. Approvals were granted by the University of Glasgow ethics committee and local education committees.

Eleven schools were invited to participate (9 in Eire; 2 in Glasgow). In each school, pupils aged at least 16 years of age were invited to participate in the study. Pupil's thoughts and attitudes were sought using word-association 'spider diagrams', using the hub-terms 'overweight', 'obese' and 'fat', each offered in a random order. Subjects wrote down their immediate word-associations, linked to each hub-term centred on an A4 sheet. Self reported data were requested on pupil's age, gender, height, and weight and clothes size.

Assessment of spider diagrams used a RUBRIC scoring cube, comprising 10 terms agreed internally by the researchers to categorise the responses and determine frequency of use. Mind-maps, drawn using Mind-map Manager (Softtomic.com), identified central themes from which secondary and tertiary themes materialised. BMI centiles were calculated using UK90 growth charts and NHS healthy weight calculator.

Eight of the 11 invited schools participated and 247 pupils (101 male) mean age 16.2 years (SD 0.5) were recruited. 98 subjects were Scottish. BMI could not be calculated for $16 \%$ of pupils who did not report height and weight. For $59 \%$ of those with data, BMI was within the healthy range and $25 \%$ of participants were categorised as overweight/obese.

About a third of responses to the three hub-terms related to poor health. 'fat' and 'overweight' were related to 'heart problem', 'diabetes' and general 'illness' by $15-16 \%$, while 'obesity' was related to these responses by $60 \%$. Close to $50 \%$ of pupils associated all three terms to "no exercise,' 'unfit, 'slow', 'poor at sport', 'TV watching”. Derogatory descriptors such as 'lazy', 'ugly', 'lack of willpower' and 'smelly' comprised $30 \%$ of responses. All hub-terms elicited responses such as 'bad', 'stupidity', 'unmotivated', 'lazy', 'inability to change', NHS and wheelchairs. Responses associated with appearance, 'heavy', 'unattractive' and 'big', were expressed by $25 \%$ of pupils. Fewer than $2 \%$ of responses expressed positive associations, including 'happy', 'funny' and 'jolly. Responses indicating a need for help with diet and activity were reported by $5 \%$ of the sample. Overweight and obese participants expressed more derogatory attitudes as the terms progressed from overweight, obese to fat, though the words chosen remained similar to the underweight and normal weight categories.

Overweight/obesity is viewed negatively by adolescents across BMI categories and commonly elicits derogatory thoughts and attitudes, while some identified the need for dietary advice and increased activity. Spider diagrams proved an acceptable tool for collection of qualitative data.

Puhl R.M., Brownell K.D. (2001). Obes Res 9, 788-905. 